\title{
Indirect tolerability comparison of Deutetrabenazine and Tetrabenazine for Huntington disease
}

Daniel O. Claassen ${ }^{1 *}$, Benjamin Carroll ${ }^{2}$, Lisa M. De Boer ${ }^{3}$, Eric Wu ${ }^{4}$, Rajeev Ayyagari ${ }^{4}$, Sanjay Gandhi ${ }^{2}$ and David Stamler ${ }^{3}$

\begin{abstract}
Background: Vesicular monoamine transporter 2 (VMAT2) inhibitors can improve hyperkinetic movements, and are effective treatment options for chorea of Huntington disease (HD). Tetrabenazine was assessed for treating chorea in the TETRA-HD trial, and while efficacious, there are tolerability concerns possibly due to its pharmacokinetic properties. Deutetrabenazine is a novel VMAT2 inhibitor that contains deuterium, which extends active metabolite half-lives and minimizes drug concentration fluctuations. In the First-HD trial, deutetrabenazine was efficacious in treating chorea and was generally well tolerated. In the absence of a head-to-head trial, we performed an indirect treatment comparison (ITC) of the tolerability of deutetrabenazine and tetrabenazine for the treatment of HD-associated chorea, as observed in the First-HD and TETRA-HD trials, using well-established comparison methods.
\end{abstract}

Methods: Data from the Phase III, 12-week, parallel-group, clinical trials First-HD $(N=90)$ and TETRA-HD $(N=84)$ were used to conduct an ITC of the tolerability of deutetrabenazine versus tetrabenazine using two anchor-based methods: Bucher comparison for unadjusted ITCs, and matching indirect comparison for adjusted ITCs. Overall adverse events (AES; mild, moderate, and severe), serious AEs, specific AEs occurring in $\geq 10 \%$ of patients, and discontinuations (all-cause and AE-related) were included in the analysis. The risk differences of these outcomes for deutetrabenazine and tetrabenazine were estimated by subtracting the applicable placebo-adjusted risk in First-HD from that of TETRA-HD. Sensitivity analyses were performed to address differences between trials, and $p$-values were obtained from z-tests.

Results: Compared with tetrabenazine, deutetrabenazine was associated with a significantly lower risk of moderate to severe AEs and neuropsychiatric AEs including agitation, akathisia, depression, depression/agitated depression, drowsiness/somnolence, insomnia, and parkinsonism in both adjusted and unadjusted analyses $(p<0.05$ for each). Deutetrabenazine had a significantly lower rate of dose reduction or dose reduction/suspension in the unadjusted and adjusted analyses ( $p<0.001$ for each). Deutetrabenazine resulted in numerically more mild AEs, such as diarrhea and coughing; however, these results were not statistically significant.

Conclusions: This indirect treatment comparison demonstrates that for the treatment of HD chorea, deutetrabenazine has a favorable tolerability profile compared to tetrabenazine.

Trial registration: ClinicalTrials.gov NCT01795859 and NCT00219804.

Keywords: Deutetrabenazine, Tetrabenazine, Huntington disease, Indirect treatment comparison, Risk difference, Huntington's chorea, Chorea, Safety, Tolerability, Movement disorders

\footnotetext{
* Correspondence: daniel.claassen@vanderbilt.edu

${ }^{1}$ Vanderbilt University, 1161 21st Avenue South A-0118, Nashville, TN 37232,

USA

Full list of author information is available at the end of the article
} International License (http://creativecommons.org/licenses/by/4.0/), which permits unrestricted use, distribution, and reproduction in any medium, provided you give appropriate credit to the original author(s) and the source, provide a link to the Creative Commons license, and indicate if changes were made. The Creative Commons Public Domain Dedication waiver (http://creativecommons.org/publicdomain/zero/1.0/) applies to the data made available in this article, unless otherwise stated. 


\section{Background}

Huntington disease (HD) is a neurodegenerative disorder characterized by chorea and progressive motor, cognitive, and behavioral symptoms $[1,2]$. The only US Food and Drug Administration (FDA)-approved drug for chorea associated with $\mathrm{HD}$ is tetrabenazine, a vesicular monoamine transporter 2 (VMAT2) inhibitor [3]. Tetrabenazine was evaluated in TETRA-HD, and while improving chorea, the adverse event (AE) profile raised tolerability concerns [3, 4].

Deutetrabenazine is a novel VMAT2 inhibitor structurally related to tetrabenazine, but it contains deuterium $[5,6]$, a naturally occurring, nontoxic form of hydrogen [7], that confers important metabolic advantages compared to tetrabenazine but does not change its target pharmacology. The introduction of deuterium in this compound attenuates drug metabolism and prolongs plasma half-life, resulting in more-uniform systemic exposure (i.e. reducing plasma fluctuations) [5, 6]. Compared with tetrabenazine, the differentiated pharmacokinetic properties have potential to improve the benefit-risk profile for patients. In First-HD, deutetrabenazine significantly reduced chorea and was generally well tolerated in patients with HD [5]. Incidences of most neuropsychiatric AEs were similar to or lower than those in the placebo group [5].

Deutetrabenazine and tetrabenazine have not been compared directly; however, because First-HD and TETRAHD have very similar study designs and were performed by the same study consortium (the Huntington Study Group), we used well-established comparative effectiveness models $[8,9]$ to compare the tolerability profiles of the two medications before and after adjusting for any putative clinical differences in populations. In this analysis, $\mathrm{AE}$ differences were assessed before and after adjusting for cross-trial differences in baseline characteristics using the Bucher method for unadjusted analyses [8], and a matching-adjusted indirect comparison method for adjusted analyses. Given the AE profile observed in First$\mathrm{HD}$, we hypothesized that tolerability of deutetrabenazine was more favorable than that of tetrabenazine.

\section{Methods}

\section{Trial comparability}

This was a retrospective study using previously reported clinical data. Full details of the First-HD (NCT01795859) and TETRA-HD (NCT00219804) study methods can be found in their respective primary reports $[3,5]$. The Phase III First-HD and TETRA-HD trials were compared closely in terms of trial design, concomitant medication use, patients' baseline characteristics, and definitions of safety outcomes. In both studies, patients underwent congruent titration periods, maintenance phases, during which dose was held stable, and 1-week washout periods (Fig. 1). The total treatment period in both studies was 12 weeks $[3,5]$.
Key First-HD inclusion criteria included manifest HD (CAG repeat length $\geq 37$ ), ambulatory, total functional capacity (TFC) score $\geq 5$, and TMC score $\geq 8$ at screening and baseline [5]. In TETRA-HD, inclusion criteria included manifest HD (CAG repeat length $\geq 37$ ), ambulatory, TFC score $>5$, and total maximal chorea (TMC) score $\geq 10$ [3]. Exclusion criteria for both trials included disabling depression, dysphagia, or dysarthria $[3,5]$. Stable treatment (for at least 8 weeks) with antidepressant medication was allowed in both trials $[3,5]$. Prior use of tetrabenazine and current treatment with dopamine D2 receptor antagonists and drugs that prolong QT intervals were exclusionary in both trials (escitalopram and citalopram allowed in First-HD). There was a higher screen failure rate in First-HD, and this was driven by (a) ensuring patients were not taking tetrabenazine to avoid selecting patients with good tolerability to tetrabenazine (N.B. the First-HD protocol allowed patients who had received tetrabenazine but only if they discontinued at least 6 months prior to screening), (b) patients were excluded if the score on the Swallowing Disturbance Questionnaire was above a cut-off established for Parkinson's disease [10], and (c) an independent functional capacity assessment was performed for all First-HD patients.

\section{Patients' baseline characteristics}

Baseline demographic and clinical differences between the First-HD and TETRA-HD cohorts were identified. Based on available sample sizes in each trial, we performed multivariable adjustments for up to three differing baseline characteristics between the two trials; age, TFC score, and TMC score. We considered these variables most clinically relevant for adjustment. Based on the review of these two cohorts, we determined that controlling for age, TFC score, and TMC was important given the potential that baseline cohort differences could impact medication tolerability, where advancing age [11], reduced TFC, and increased TMC may portend a more-advanced disease with increased likelihood of developing certain AEs.

\section{Indirect comparison}

Tetrabenazine data were collected from the published clinical trial results and from the FDA-approved prescribing information [3, 12]. Aggregate data from TETRA-HD and patient-level data from First-HD were used to conduct an indirect comparison of AEs using anchor-based methodology. For unadjusted results, the Bucher method was used to compare two placebo-controlled trials with different comparators and to calculate a summary risk difference of the two treatments (i.e. tetrabenazine and deutetrabenazine) [8]. The Bucher method is a well-established and accepted analytical method used across disease states for conducting indirect comparisons [13-16]. 


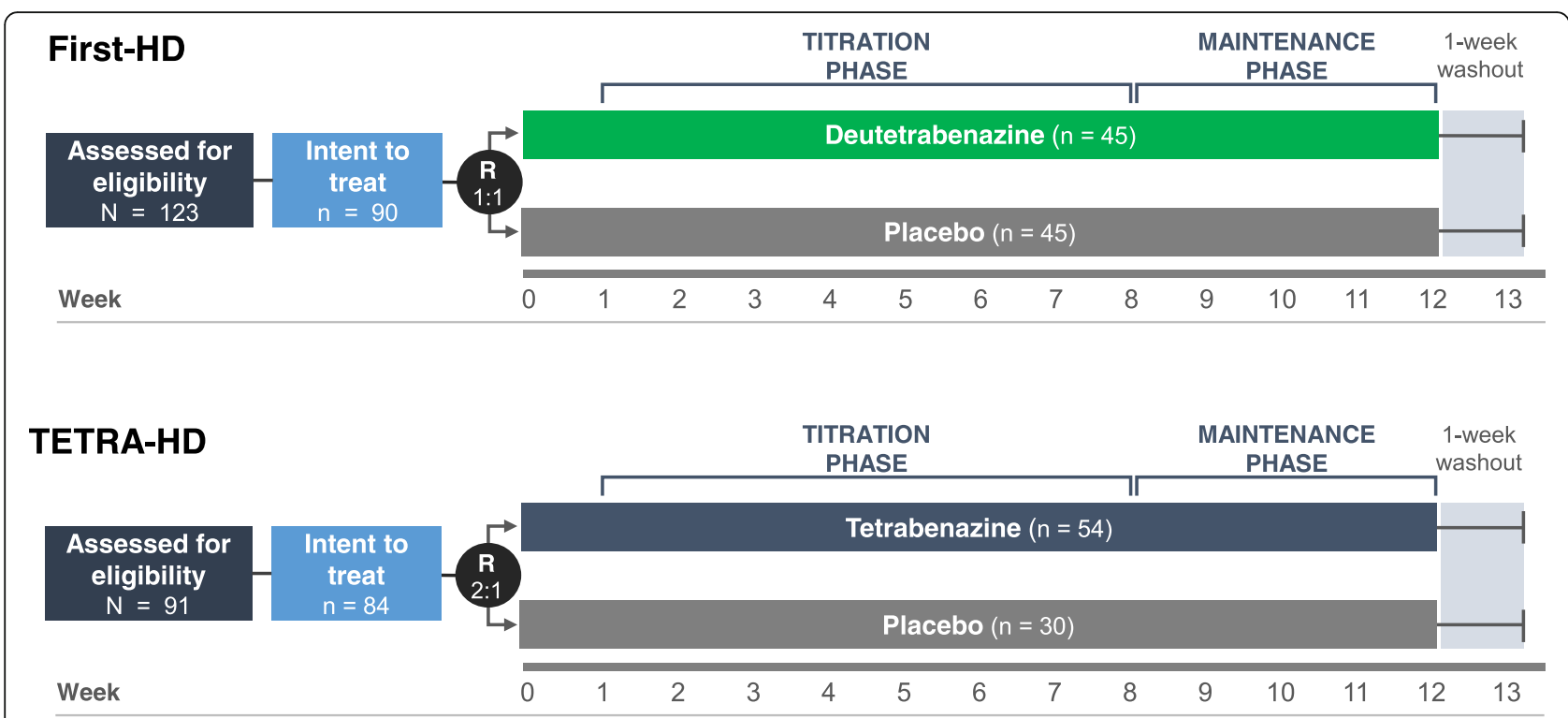

Fig. 1 First-HD and TETRA-HD study designs. The First-HD and TETRA-HD study designs were very similar. For both studies, dose adjustments were allowed up to and including Week 7, followed by a maintenance period and a 1-week washout for both studies. $R=$ randomization ratio

A matching-adjusted indirect comparison was used to evaluate $\mathrm{AE}$ outcomes between patients treated with deutetrabenazine or tetrabenazine, which provided the ability to account for differences in the baseline characteristics of the two patient populations. This more sophisticated ITC method incorporates a propensity score model, which estimates the effect of a treatment, accounting for baseline clinical covariates that predict a treatment response. Thus, the propensity score of individual patient data from one trial is weighted such that the population resembles that of the comparator trial [9]. After this weighting adjustment, a Bucher-type comparison is then applied across the adjusted populations as a part of the matching-adjusted indirect comparison method. In this analysis, the First-HD patient baseline characteristics were weighted to resemble the TETRAHD patients more closely.

Both methods provided estimates for the within-trial risk difference of deutetrabenazine and tetrabenazine for each safety outcome. Within-trial risk differences were calculated for each trial as the incidence of AEs in the active arm minus that in the placebo arm of the same trial. The summary risk differences between deutetrabenazine and tetrabenazine were estimated by subtracting the within-trial risk differences of TETRA-HD from the respective within-trial risk differences of First-HD. Number needed to harm $(\mathrm{NNH})$ estimates were defined as the average number of patients that need to be treated with tetrabenazine instead of deutetrabenazine for an average of one additional patient to experience an AE. $\mathrm{NNH}$ estimates were calculated as the reciprocal of the summary risk differences.

\section{Safety outcomes}

Any AE, moderate to severe AEs, mild AEs, serious AEs (SAEs), discontinuations (all-cause and AE-related), dose reductions, and specific AEs that occurred in $\geq 10 \%$ of patients were included in the analysis. An incidence rate of $10 \%$ was deemed clinically significant.

\section{Sensitivity analyses}

In addition to the previously described analyses, a matching-adjusted indirect comparison was performed with adjustments for different combinations of baseline characteristics (age, TFC score, and TMC) to test the robustness of the findings. Also, AEs were coded according to Medical Dictionary for Regulatory Activities (MedDRA) and World Health Organization (WHO) Preferred Terms in First-HD and TETRA-HD, respectively. AEs reported in the tetrabenazine FDA-approved prescribing information were examined and compared with those reported in the primary TETRA-HD publication $[3,12]$. Whenever ambiguity in definitions existed due to potential $\mathrm{AE}$ coding differences between the two trials, multiple versions of the First-HD AE definition were used within the analysis to assess the potential for any bias.

\section{Statistical analyses}

Baseline characteristics and AEs from TETRA-HD [3] were summarized and compared to pre-existing summaries in the First-HD clinical study report. Baseline characteristics in the respective arms (i.e. active vs active and placebo vs placebo) for each trial were compared using chi-squared analysis for categorical variables and t-tests for continuous variables. $P$-values for risk differences were 
obtained using z-tests. Versions 9.2 and 9.3 of the SAS statistical software package were used for all analyses (SAS Institute, Inc.).

\section{Results}

\section{Comparison of patients}

While there were statistical differences between the overall populations enrolled in each clinical trial, the distributions for most variables were similar. Mean baseline TMC scores for patients enrolled in First-HD and TETRA-HD were 12.7 and 14.9, respectively [3]. Before adjustment, patients in the deutetrabenazine arm of First-HD had significantly higher ages, as well as TFC score, verbal fluency, symbol digit and Stroop word reading, behavioral assessment, functional checklist, independence scale and Barnes Akathisia Rating Scale (BARS) scores compared with the tetrabenazine arm of TETRA-HD (Table 1). In addition, patients in the deutetrabenazine arm in First-HD had significantly lower CAG repeat lengths as well as parkinsonism, TMS, and Unified Parkinson's Disease Rating Scale (UPDRS) speech scores. The placebo arms in each trial only differed in gender, TMC score, and BARS score before weighting. These differences were further reduced after weighting.

\section{Comparison of safety outcomes}

Before and after adjustment, deutetrabenazine demonstrated significantly lower incidence rates for overall AEs and moderate to severe AEs than tetrabenazine $(p<0.001$ for each; Table 2). This resulted in a significantly lower risk for moderate to severe AEs with deutetrabenazine compared with tetrabenazine in the unadjusted $(-39.6 \%$, 95\% CI: $-67.1,-12.2 \% ; p=0.005)$ and adjusted $(-46.4 \%$, 95\% CI: $-79.4,-13.3 \% ; p=0.006$ ) analyses (Fig. 2, Table 3). Although not statistically significant, deutetrabenazine resulted in a greater risk of mild AEs in the unadjusted (18.9\%, 95\% CI: $-9.6 \%, 47.4 \% ; p=0.194)$ and adjusted (11.1\%, 95\% CI: $-24.4 \%, 46.6 \%$; $p=0.540)$ analyses, specifically with a greater incidence of coughing (before adjustment $p=0.062$, after adjustment $p=0.040$ ) and diarrhea (before adjustment $p=0.788$, after adjustment $p=0.951$ ). Overall dose reductions and dose reductions/suspensions due to AEs occurred significantly less frequently with deutetrabenazine compared with tetrabenazine before and after placebo adjustment ( $p<0.001$ for all comparisons).

Deutetrabenazine demonstrated a significantly lower incidence of several individual AEs compared with tetrabenazine (Table 2). Before adjustment, patients treated with deutetrabenazine had significantly lower incidence of agitation, akathisia, anxiety, depression, drowsiness/somnolence, fatigue, insomnia, and parkinsonism compared with those treated with tetrabenazine ( $p<0.05$ for each). After adjustment, in addition to the AEs mentioned above, deutetrabenazine demonstrated significantly lower incidences of depression/agitated depression, falls, and nausea $(p<0.05$ for each). Before adjustment, there was significantly lower risk for agitation $(-12.6 \%, 95 \% \mathrm{CI}:-23.0,-2.2 \% ; p=0.018)$, akathisia $(-18.5 \%, 95 \%$ CI: $-30.5,-6.5 \% ; p=0.003)$, depression (-19.3\%, 95\% CI: $-32.0,-6.6 \% ; p=0.003)$, depression/agitated depression $(-17.0 \%, 95 \%$ CI: $-30.4, \quad-3.7 \% ; \quad p=0.013), \quad$ drowsiness/somnolence $(-21.5 \%, 95 \%$ CI: $-39.2,-3.7 \% ; p=0.018)$, insomnia $(-23.7 \%, 95 \%$ CI: $-38.7,-8.7 \%$; $p=0.002)$, and parkinsonism $(-14.8 \%, 95 \%$ CI: $-24.3,-5.3 \%$; $p=0.002)$ with deutetrabenazine treatment compared with tetrabenazine treatment (Fig. 3, Table 3). After adjustment, the risk for the following AEs remained significantly lower for deutetrabenazine compared with tetrabenazine: agitation $(-14.2 \%$, 95\% CI: $-24.6,-3.8 \%$; $p=0.007)$, akathisia $(-18.9 \%$, 95\% CI: $-32.0,-5.8 \% ; p=0.005)$, depression $(-20.8,95 \% \mathrm{CI}:-33.8$, $-7.8 \% ; p=0.002)$, depression/agitated depression $(-20.2 \%$, 95\% CI: $-33.9,-6.5 \% ; p=0.004)$, drowsiness/somnolence $(-22.9 \%, 95 \%$ CI: $-44.9,-0.8 \% ; \quad p=0.042)$, insomnia $(-24.3 \%, 95 \%$ CI: $-40.9,-7.6 ; p=0.004)$, parkinsonism $(-14.8 \%, 95 \%$ CI: $-24.3,-5.3 \% ; p=0.002)$. The risk for other AEs did not significantly differ between treatment groups; however, deutetrabenazine was associated with numerically lower risk of most of these AEs compared with tetrabenazine, except for coughing and diarrhea. To test whether there was any effect of multiple comparisons on the statistically significant individual $\mathrm{AE}$ findings, we performed the Benjamini-Hochberg false discovery rate controlling procedure at the 0.1 level. The statistical significance of the results remained unchanged in both the unadjusted and adjusted analyses.

Deutetrabenazine-treated patients had significantly lower risk for dose reduction or dose reduction/suspension compared with those treated with tetrabenazine in the unadjusted (-41.1\%, 95\% CI: $-59.1,-23.1 \%$; $p<0.001$ for reduction and $-41.1 \%, 95 \% \mathrm{CI}:-60.0,-22.3 \%$; $p<0.001$ for reduction/suspension) and adjusted $(-40.5 \%, 95 \% \mathrm{CI}$ : $-62.0,-19.0 \% ; p<0.001$ for dose reduction and $-41.6 \%$, 95\% CI: $-63.9,-19.3 \% ; p<0.001$ for dose reduction/suspension) analyses (Fig. 4, Table 3). The incidence of discontinuations due to AEs was numerically lower with deutetrabenazine versus tetrabenazine in the unadjusted analysis $(p=0.144)$, and was significantly lower with deutetrabenazine compared with tetrabenazine in the adjusted analysis $(p=0.030)$. While there was a trend toward lower risk for discontinuations due to AEs with deutetrabenazine compared with tetrabenazine before adjustment $(-9.3 \%$, 95\% CI: $-19.1,0.6 \% ; p=0.065)$, discontinuations due to AEs in deutetrabenazine patients were significantly lower after adjustment $(-10.4 \%$, 95\% CI: $-20.3,-0.4 \% ; p=0.041)$ (Fig. 4, Table 3).

$\mathrm{NNH}$ values were statistically significantly favorable for deutetrabenazine compared with tetrabenazine for moderate to severe AEs in the unadjusted analysis $(\mathrm{NNH}=3,95 \%$ CI: 1,8$)$, and adjusted analysis $(\mathrm{NNH}=2,95 \% \mathrm{CI}: 1,7)$. 


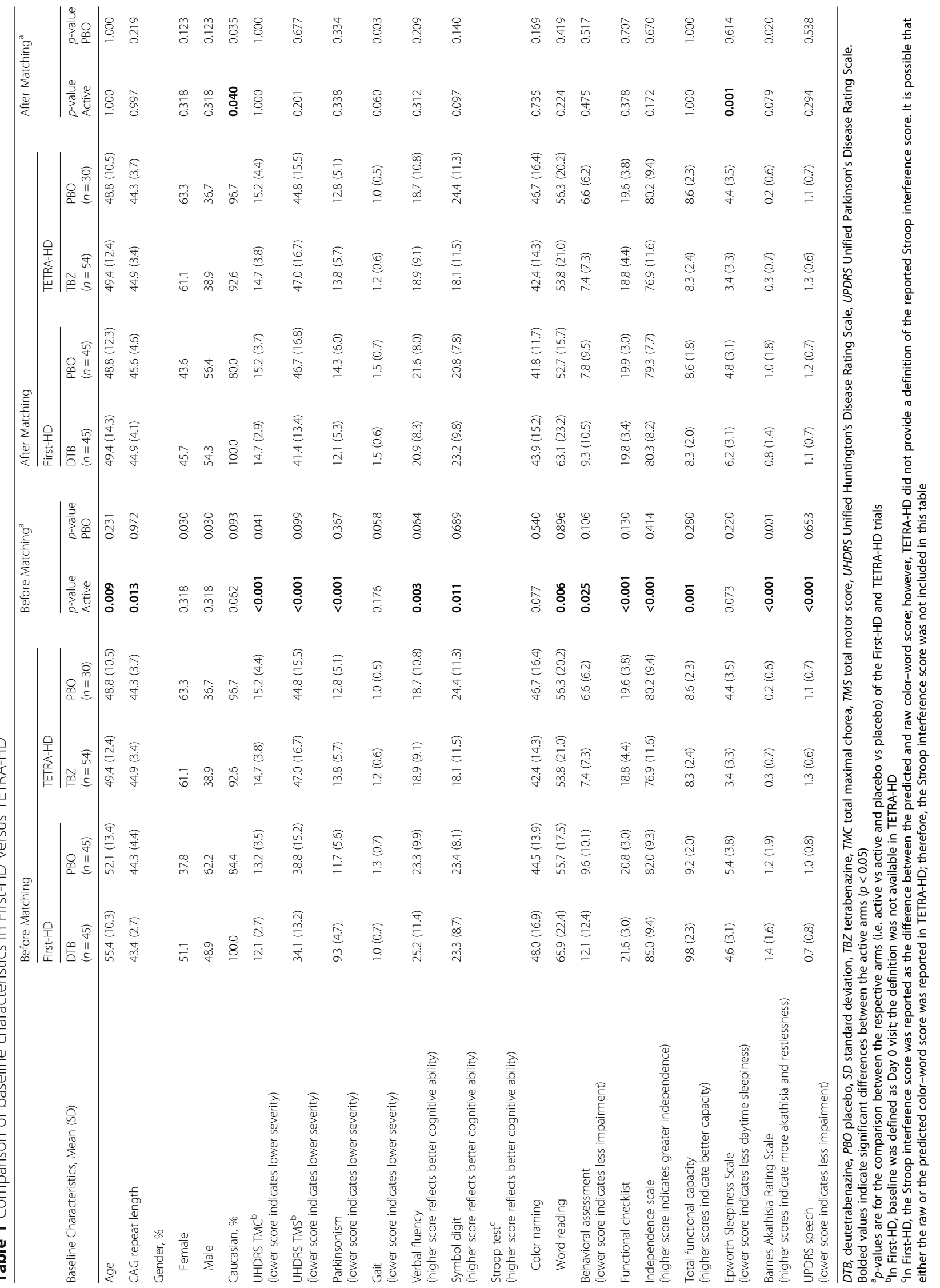




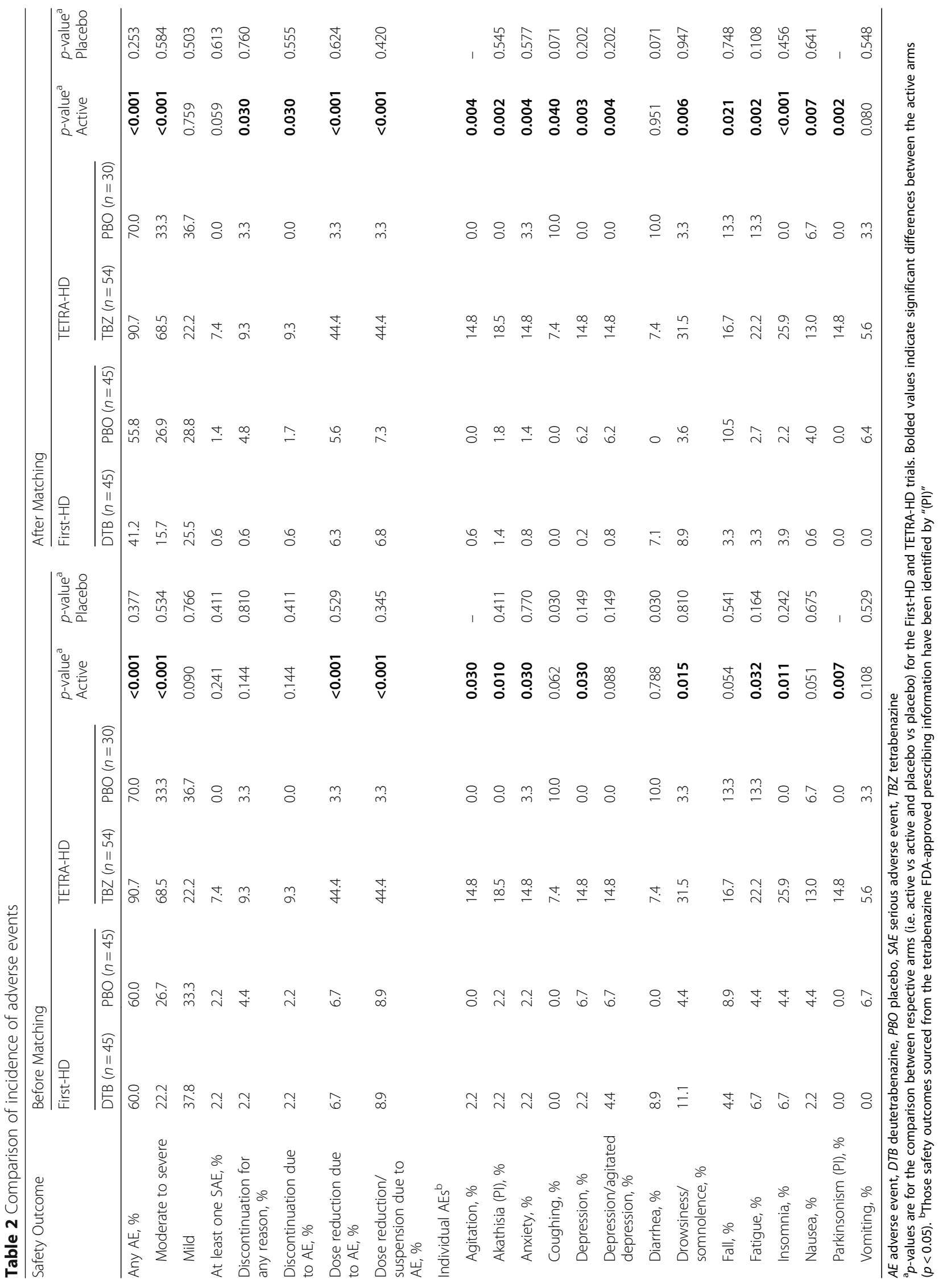




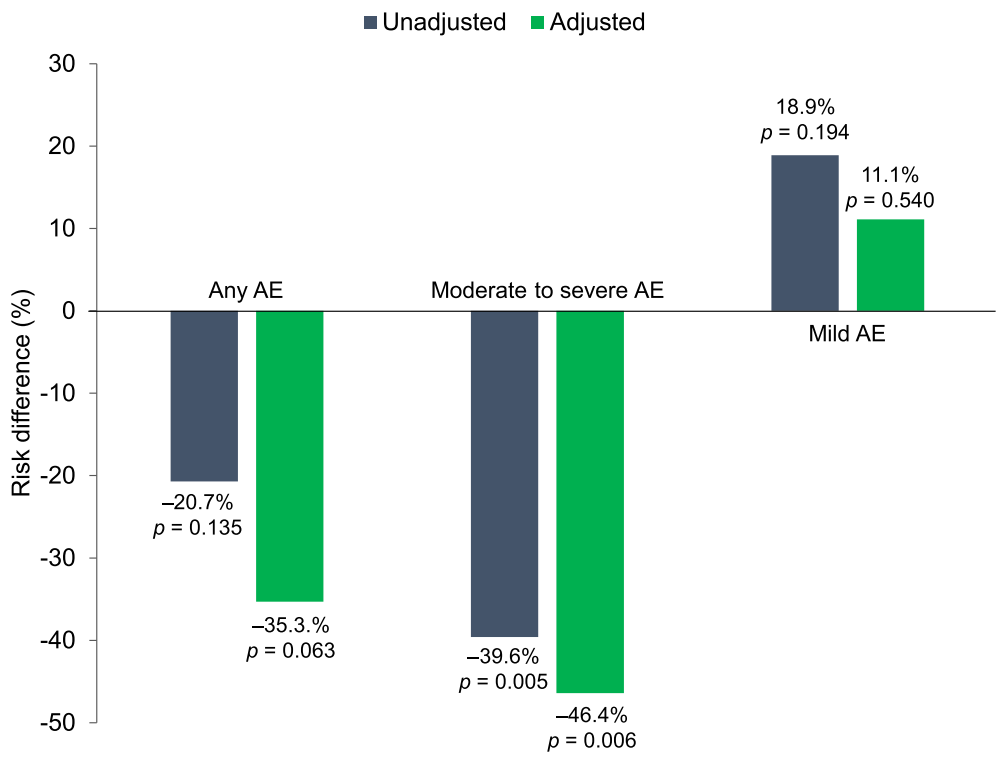

Fig. 2 Risk differences for adverse events by severity. The risk differences were assessed before and after placebo-adjustment. Negative risk difference favors deutetrabenazine. $p$-values comparing the risk differences between deutetrabenazine and tetrabenazine were obtained from $z$-tests. AE $=$ adverse event

That is, after adjustment, if two patients were treated with tetrabenazine instead of deutetrabenazine, one more patient, on average, would experience a moderate to severe $\mathrm{AE}$. Likewise, $\mathrm{NNH}$ were significantly favorable for deutetrabenazine compared with tetrabenazine for the following adverse events in both adjusted and unadjusted analyses: agitation $(\mathrm{NNH}=8$ [95\% CI: 4, 46] unadjusted; $\mathrm{NNH}=7$ [95\% CI: 4, 26] adjusted); akathisia (NNH $=5$ [95\% CI: 3 , 15] unadjusted; $\mathrm{NNH}=5$ [95\% CI: 3, 17] adjusted); depression $(\mathrm{NNH}=5$ [95\% CI: 3, 15] unadjusted; $\mathrm{NNH}=5$ [95\% CI: 3, 13] adjusted); depression/agitated depression $(\mathrm{NNH}=6$ [95\% CI: 3, 27] unadjusted; $\mathrm{NNH}=5$ [95\% CI: $3,15]$ adjusted); drowsiness/somnolence ( $\mathrm{NNH}=5[95 \%$ CI: 3, 27] unadjusted; $\mathrm{NNH}=4$ [95\% CI: 2, 119] adjusted); insomnia $(\mathrm{NNH}=4$ [95\% CI: 3, 12] unadjusted; $\mathrm{NNH}=4$ [95\% CI: 2, 13] adjusted); and parkinsonism $(\mathrm{NNH}=7$ [95\% CI: 4, 19] for both). No other AEs analyzed were statistically significant.

\section{Sensitivity analyses}

There were no meaningful changes in the results with different combinations of baseline characteristics included for adjustments in the matching-adjusted indirect comparisons (Fig. 3). Results based on the tetrabenazine FDA approved prescribing information were similar to those based on the TETRA-HD publication [3, 12]. Results for depression and dose reduction due to AEs were not sensitive to different definitions used by each trial (Table 3).

\section{Discussion}

Results of this comparative analysis emphasize that compared to tetrabenazine, patients treated with deutetrabenazine experienced a significantly lower risk for moderate to severe AEs and dose reductions due to AEs in the unadjusted and adjusted data sets. Discontinuations were also significantly lower in the adjusted data set. These results corroborate the expected improvement in the benefit-risk profile of deutetrabenazine, potentially due to the differentiated pharmacokinetic profile resulting from deuterium substitution and attenuated metabolism. This is particularly relevant because long-term evaluation of tetrabenazine use in patients with HD indicates that tetrabenazine tolerability, rather than a plateau of efficacy, is often dose-limiting [17].

The European Huntington's Disease Network (EHDN) REGISTRY study reported that $<10 \%$ of patients with HD taking medications receive tetrabenazine [18]. Clinical management of chorea is not uniform, and comorbid psychiatric comorbidities commonly seen in HD patients (e.g. delusions, behavioral outbursts, and psychosis) often necessitate the use of neuroleptics, which can also provide improvements to choreiform movements. Of course, neuroleptics also have significant side effects, such as worsening parkinsonism, metabolic derangements (among other side effects), and poor tolerability which can limit their use. However, given our ITC findings, if improved tolerability of deutetrabenazine is replicated in larger HD populations, this would potentially allow for increased options for managing chorea and titration up to higher doses, thus maximizing chorea control with fewer dose-limiting AEs [19].

We note that deutetrabenazine had a significantly lower risk for several neuropsychiatric AEs compared with tetrabenazine, including insomnia, drowsiness/ somnolence, depression, akathisia, depression/agitated 
Table 3 Summary risk difference comparison of adverse events

\begin{tabular}{|c|c|c|c|c|c|c|}
\hline \multirow[t]{2}{*}{ Safety Outcome } & \multicolumn{3}{|c|}{ Unadjusted Risk Difference } & \multicolumn{3}{|c|}{ Adjusted Risk Difference } \\
\hline & DTB vs TBZ & $95 \% \mathrm{Cl}$ & $p$-value & DTB vs TBZ & $95 \% \mathrm{Cl}$ & $p$-value \\
\hline Any $A E, \%$ & -20.7 & $-47.9,6.4$ & 0.135 & -35.3 & $-72.4,1.8$ & 0.063 \\
\hline Moderate to severe & -39.6 & $-67.1,-12.2$ & 0.005 & -46.4 & $-79.4,-13.3$ & 0.006 \\
\hline Mild & 18.9 & $-9.6,47.4$ & 0.194 & 11.1 & $-24.4,46.6$ & 0.540 \\
\hline At least one $S A E, \%$ & -7.4 & $-16.7,1.9$ & 0.117 & -8.2 & $-17.4,1.0$ & 0.080 \\
\hline Discontinuation for any reason, \% & -8.1 & $-20.6,4.3$ & 0.201 & -10.1 & $-23.4,3.2$ & 0.136 \\
\hline Discontinuation due to $A E, \%$ & -9.3 & $-19.1,0.6$ & 0.065 & -10.4 & $-20.3,-0.4$ & 0.041 \\
\hline Dose reduction due to $\mathrm{AE}$ & -41.1 & $-59.1,-23.1$ & $<0.001$ & -40.5 & $-62.0,-19.0$ & $<0.001$ \\
\hline Dose reduction/suspension due to $\mathrm{AE}$ & -41.1 & $-60.0,-22.3$ & $<0.001$ & -41.6 & $-63.9,-19.3$ & $<0.001$ \\
\hline \multicolumn{7}{|l|}{ Individual $\mathrm{AEs}^{\mathrm{a}}$} \\
\hline Agitation, \% & -12.6 & $-23.0,-2.2$ & 0.018 & -14.2 & $-24.6,-3.8$ & 0.007 \\
\hline Akathisia (PI), \% & -18.5 & $-30.5,-6.5$ & 0.003 & -18.9 & $-32.0,-5.8$ & 0.005 \\
\hline Anxiety, \% & -11.5 & $-24.4,1.5$ & 0.083 & -12.0 & $-25.2,1.2$ & 0.074 \\
\hline Coughing, $\%$ & 2.6 & $-10.2,15.4$ & 0.692 & 2.6 & $-10.2,15.4$ & 0.692 \\
\hline Depression, \% & -19.3 & $-32.0,-6.6$ & 0.003 & -20.8 & $-33.8,-7.8$ & 0.002 \\
\hline Depression/agitated depression, \% & -17.0 & $-30.4,-3.7$ & 0.013 & -20.2 & $-33.9,-6.5$ & 0.004 \\
\hline Diarrhea, \% & 11.5 & $-3.8,26.8$ & 0.141 & 9.7 & $-9.4,28.7$ & 0.320 \\
\hline Drowsiness/somnolence, \% & -21.5 & $-39.2,-3.7$ & 0.018 & -22.9 & $-44.9,-0.8$ & 0.042 \\
\hline Fall, \% & -7.8 & $-26.5,11.0$ & 0.417 & -10.6 & $-32.1,10.9$ & 0.336 \\
\hline Fatigue, \% & -6.7 & $-25.6,12.3$ & 0.491 & -8.3 & $-28.3,11.7$ & 0.416 \\
\hline Insomnia, \% & -23.7 & $-38.7,-8.7$ & 0.002 & -24.3 & $-40.9,-7.6$ & 0.004 \\
\hline Nausea, \% & -8.5 & $-23.2,6.1$ & 0.255 & -9.7 & $-24.7,5.4$ & 0.207 \\
\hline Parkinsonism (PI), \% & -14.8 & $-24.3,-5.3$ & 0.002 & -14.8 & $-24.3,-5.3$ & 0.002 \\
\hline Vomiting, \% & -8.9 & $-20.4,2.6$ & 0.129 & -8.7 & $-21.1,3.8$ & 0.173 \\
\hline
\end{tabular}

$A E$ adverse event, $C l$ confidence interval, DTB deutetrabenazine, SAE serious adverse event, $T B Z$ tetrabenazine

${ }^{a}$ Those safety outcomes sourced from the tetrabenazine FDA-approved prescribing information have been identified by "(PI)". Bolded values indicate significant risk differences between deutetrabenazine and tetrabenazine $(p<0.05)$

depression, parkinsonism, and agitation. The risk difference for anxiety and fatigue also favored deutetrabenazine, although these did not reach significance. All of the seven AEs, agitation, akathisia, depression, depression/agitated depression, drowsiness/somnolence, insomnia, and parkinsonism, remained significantly lower with deutetrabenazine treatment even after applying Benjamini-Hochberg corrections. This further highlights the robustness of these results. In HD, disease severity is often associated with the progression of psychiatric co-morbidities, which are likely secondary to the disease process [20]. Although tetrabenazine is the only FDA-approved drug for chorea associated with HD, there is concern regarding exacerbation of neuropsychiatric comorbidities (especially depression) [21], frequently encountered in patients with HD; however, treated depression is not a contraindication for its use $[21,22]$. Approximately $40 \%$ of patients with HD report significant depressive symptoms, such as low selfesteem, sadness, anxiety, and suicidal ideation [23]. The most recent American Academy of Neurology guidelines name depression and parkinsonism as the most concerning AEs related to tetrabenazine, and close monitoring of all patients is recommended [22]; depression AEs have been shown to occur in patients with and without preexisting depression [21]. The significantly lower risk for neuropsychiatric AEs with deutetrabenazine as compared with tetrabenazine, including depression and parkinsonism, suggests that deutetrabenazine offers patients an alternative treatment option that may be less likely to induce or exacerbate neuropsychiatric symptoms. The lower risks of AEs observed with deutetrabenazine may be due to the substitution of hydrogen with deuterium at specific sites, which attenuates the metabolism and results in a more stable pharmacokinetic profile.

There are potential limitations of this analysis that should be considered. Before adjustment, patients in the deutetrabenazine arm in First-HD had higher TFC scores and lower CAG repeat lengths and TMS scores, suggesting that patients treated with deutetrabenazine were less advanced in their disease than those treated 


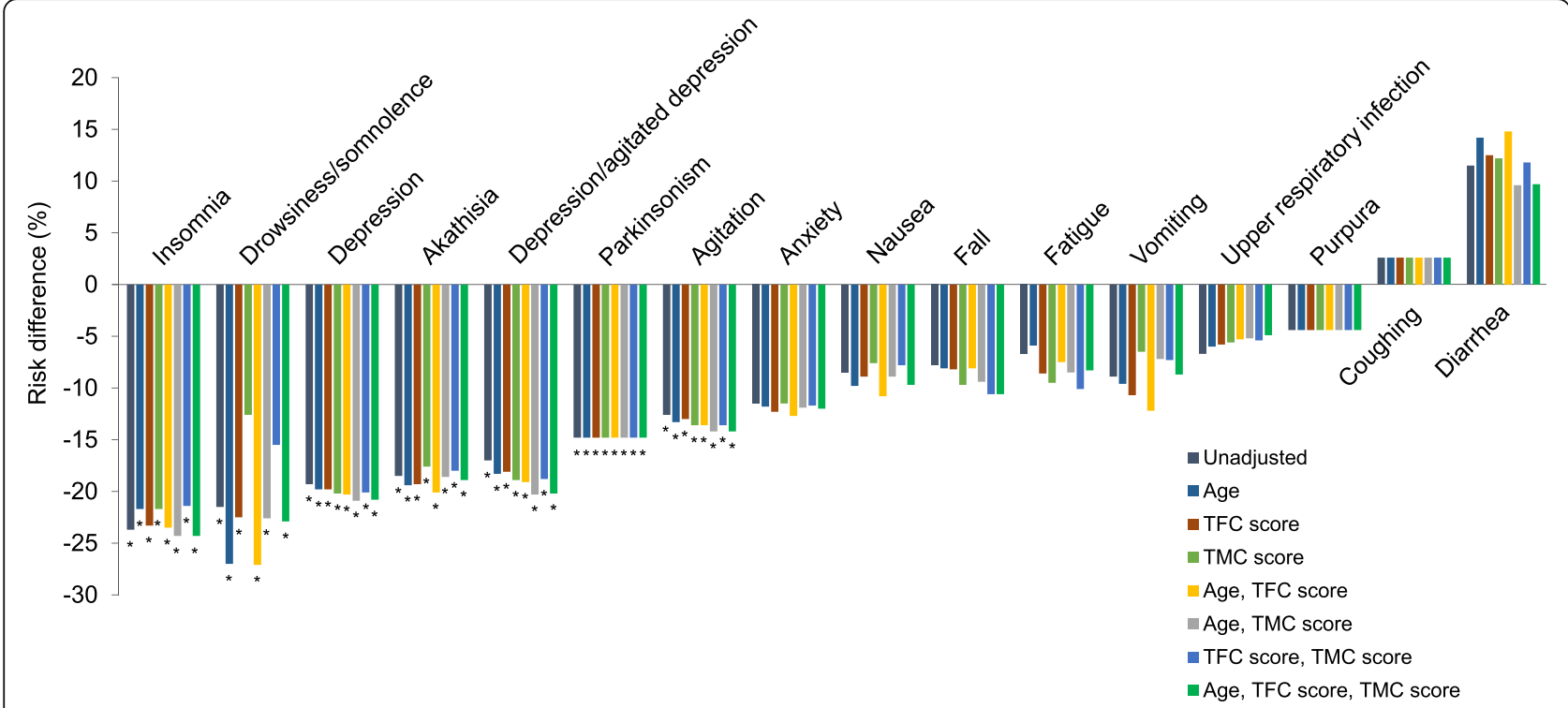

Fig. 3 Risk difference for specific adverse events. The risk differences for specific adverse events, including insomnia, drowsiness/somnolence, depression, akathisia, depression/agitated depression, parkinsonism, agitation, anxiety, vomiting, nausea, fall, fatigue, vomiting, upper respiratory infection, purpura, diarrhea, and coughing were assessed. Negative risk difference favors deutetrabenazine. The figure presents unadjusted data, in addition to data adjusted by baseline characteristics (TMC, TFC, and/or age). ${ }^{*} p<0.05$. $p$-values comparing the risk differences between deutetrabenazine and tetrabenazine were obtained from z-tests. TFC = total functional capacity, TMC = total maximal chorea

with tetrabenazine in TETRA-HD, and may have a different disease course. We suspect that lower CAG repeat length in the First-HD group was driven primarily by the recruitment of tetrabenazine-naïve patients, and less likely by the strict swallowing and functional capacity assessment criteria in the study. The analysis adjusted for important clinical differences between the First-HD and TETRA-HD patient groups. However, the sample sizes precluded all clinical and demographic variables to be included in the model. So while we were able to adjust for key clinical differences (TFC, age, and TMC), we were not able to adjust for some baseline characteristics,

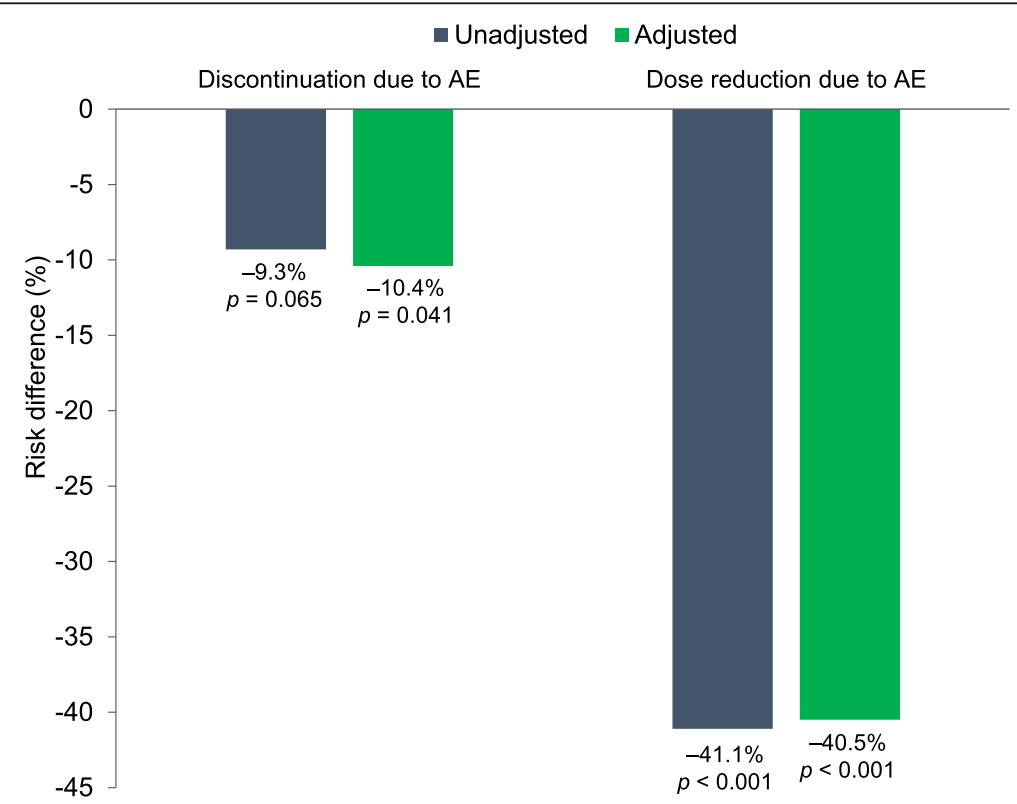

Fig. 4 Risk differences for discontinuations and dose reductions due to adverse events. The risk differences were assessed before and after placeboadjustment. Negative risk difference favors deutetrabenazine. $p$-values comparing the risk differences between deutetrabenazine and tetrabenazine were obtained from z-tests. $A E=$ adverse event 
such as CAG repeat length and TMS scores, due to sample size and model parsimony considerations. The criteria used to select which variables were to be included in the model included collinearity with other variables to achieve a robust adjustment of differences between study groups. As with any indirect comparison, the matching-adjusted indirect comparison method cannot adjust for differences in patient characteristics that were not observed, or not reported in one of the trials. While $\mathrm{AE}$ definitions were generally analogous, they could be compared directly only when the TETRA-HD publication reported the definition. In addition, sample sizes in the trials only permitted adjustment for up to three population characteristics at a time; however, the results remained largely unchanged regardless of characteristics included for adjustment.

\section{Conclusions}

In both adjusted and unadjusted comparisons, deutetrabenazine demonstrated significantly lower rates of both aggregated and individual safety outcomes compared with tetrabenazine. This is especially important in patients with HD, who may have a high disease burden and several neuropsychiatric comorbidities. First-HD and TETRA-HD had similar inclusion/exclusion criteria, protocols, patient populations, study endpoints, and interventions, and therefore required limited adjustment for matching baseline data. Moreover, the clinical sites were similar in experience and training. Because of this, the outcomes were consistent across sensitivity analyses even after modifying the definitions of AEs, data sources (primary publication vs FDA-approved prescribing information), and adjustments for different baseline characteristics. These results reinforce the favorable safety profile observed during the First-HD study, which may be attributed to the differentiated pharmacokinetic profile of deutetrabenazine resulting from deuterium substitution. Taken together, these data suggest that deutetrabenazine may be a well-tolerated treatment option for patients with chorea associated with HD, and may allow titration to an optimal therapeutic dose with fewer treatment disruptions due to AEs. The potential benefit of this optimized therapy is that greater efficacy, i.e., chorea control, may be achieved which may in turn lead to improved function [5]. Future clinical trials of deutetrabenazine are needed to confirm long-term clinical benefits of deutetrabenazine and patient persistence on treatment. In addition, a head to head trial would provide the best evidence for comparison of tolerability between the two medications.

\footnotetext{
Abbreviations

AE: Adverse event; BARS: Barnes Akathisia Rating Scale; Cl: Confidence interval; EHDN: European Huntington's Disease Network; FDA: US Food and Drug Administration; HD: Huntington disease; MedDRA: Medical Dictionary for Regulatory Activities; NNH: Number needed to harm; SAE: Serious adverse event; TFC: Total functional capacity; TMC: Total maximal chorea; TMS: Total motor score; UHDRS: Unified Huntington's Disease Rating Scale; UPDRS: Unified
}

Parkinson's Disease Rating Scale; VMAT2: Vesicular monoamine transporter 2; WHO: World Health Organization

\section{Acknowledgments}

We thank the patients and site personnel involved with both trials, and Arianna Grove, PhD (Chameleon Communications International with funding from Teva Pharmaceutical Industries), for editorial assistance in the preparation of this report. We would also like to thank Ravi lyer, PhD, MBA, an employee of Teva

Pharmaceutical Industries, for his contributions to the study concept and analyses. Statistical analysis conducted by: Rajeev Ayyagari of Analysis Group, Inc., Boston, Massachusetts, USA.

\section{Funding}

This study was funded by Teva Pharmaceutical Industries, Petach Tikva, Israel.

Availability of data and materials

All relevant data generated or analyzed during this study are included in this published article.

\section{Authors' Contributions}

DOC: manuscript preparation, significant content-related direction and feedback on all relevant materials throughout the development of the manuscript. BC: significant content-related direction and feedback on all relevant materials throughout the development of the manuscript. LMD: significant contentrelated direction and feedback on all relevant materials throughout the development of the manuscript. EW: significant content-related direction and feedback on all relevant materials throughout the development of the manuscript. RA: significant content-related direction and feedback on all relevant materials throughout the development of the manuscript. SG: significant content-related direction and feedback on all relevant materials throughout the development of the manuscript. DS: significant contentrelated direction and feedback on all relevant materials throughout the development of the manuscript. All authors read and approved the final manuscript.

\section{Competing interests}

DOC: Grant support: NIH/NINDS, Michael J Fox Foundation, Huntington Disease Society of America, Vaccinex, AbbVie, Auspex Pharmaceuticals. Consulting fees: Teva Neuroscience, Lundbeck, Acadia, AbbVie. BC: Employee of Teva Pharmaceutical Industries. LMD: Former employee of Teva Pharmaceutical Industries. EW: Employee of Analysis Group, which received consulting fees from Teva Pharmaceutical Industries for the conduct of this study. RA: Employee of Analysis Group, which received consulting fees from Teva Pharmaceutical Industries for the conduct of this study. SG: Employee of Teva Pharmaceutical Industries. DS: Employee of Teva Pharmaceutical Industries.

Consent for publication

Not applicable.

Ethics approval and consent to participate

Not applicable.

\section{Author details}

'Vanderbilt University, 1161 21st Avenue South A-0118, Nashville, TN 37232, USA. ${ }^{2}$ Teva Pharmaceuticals, Frazer, PA, USA. ${ }^{3}$ Teva Pharmaceuticals, La Jolla, CA, USA. ${ }^{4}$ Analysis Group, Inc., Boston, MA, USA.

Received: 10 November 2016 Accepted: 2 February 2017

Published online: 01 March 2017

\section{References}

1. Ross CA, Aylward EH, Wild EJ, et al. Huntington disease: natural history, biomarkers and prospects for therapeutics. Nat Rev Neurol. 2014:10:204-16.

2. Walker FO. Huntington's disease. Semin Neurol. 2007;27:143-50.

3. Huntington Study Group. Tetrabenazine as antichorea therapy in Huntington disease: a randomized controlled trial. Neurology. 2006;66:366-72.

4. Jankovic J, Roos RAC. Chorea associated with Huntington's disease: to treat or not to treat? Mov Disord. 2014;29:1414-8.

5. Huntington Study Group, Frank S, Testa CM, et al. Effect of deutetrabenazine on chorea among patients with Huntington disease: a randomized clinical trial. JAMA. 2016;316:40-50. 
6. Stamler D, Bradbury M, Brown F. The pharmacokinetics and safety of deuterated-tetrabenazine. Neurology. 2013;80:P07. 210.

7. Shao L, Hewitt MC. The kinetic isotope effect in the search for deuterated drugs. Drug News Perspect. 2010;23:398-404.

8. Bucher HC, Guyatt GH, Griffith LE, Walter SD. The results of direct and indirect treatment comparisons in meta-analysis of randomized controlled trials. J Clin Epidemiol. 1997;50:683-91.

9. Signorovitch J, Erder MH, Xie J, et al. Comparative effectiveness research using matching-adjusted indirect comparison: an application to treatment with guanfacine extended release or atomoxetine in children with attention-deficit/hyperactivity disorder and comorbid oppositional defiant disorder. Pharmacoepidemiol Drug Saf. 2012;21(2):130-7.

10. Cohen JT, Manor Y. Swallowing disturbance questionnaire for detecting dysphagia. Laryngoscope. 2011;121(7):1383-7.

11. Kinirons MT, O'Mahony MS. Drug metabolism and ageing. Br J Clin Pharmacol. 2004;57:540-4.

12. XENAZINE Prescribing Information. Lundbeck, Inc. 2015. p.1-23.

13. Cucherat M, Stalmans I, Rouland JF. Relative efficacy and safety of preservativefree latanoprost (T2345) for the treatment of open-angle glaucoma and ocular hypertension: an adjusted Indirect comparison meta-analysis of randomized clinical trials. J Glaucoma. 2014;23:e69-75.

14. Fenix-Caballero S, Alegre-del Rey EJ, Castano-Lara R, Puigventos-Latorre F, Borrero-Rubio JM, Lopez-Vallejo JF. Direct and indirect comparison of the efficacy and safety of adalimumab, etanercept, infliximab and golimumab in psoriatic arthritis. J Clin Pharm Ther. 2013;38:286-93.

15. Galvan-Banqueri M, Marin Gil R, Santos Ramos B, Bautista Paloma FJ. Biological treatments for moderate-to-severe psoriasis: indirect comparison. J Clin Pharm Ther. 2013;38:121-30.

16. Zagmutt FJ, Tarrants ML. Indirect comparisons of adverse events and dropout rates in early Parkinson's disease trials of pramipexole, ropinirole, and rasagiline. Int J Neurosci. 2012;122:345-53.

17. Shen V, Clarence-Smith K, Hunter C, Jankovic J. Safety and Efficacy of Tetrabenazine and Use of Concomitant Medications During Long-Term, Open-Label Treatment of Chorea Associated with Huntington's and Other Diseases. Tremor Other Hyperkinet Mov (N Y). 2013;3.

18. Orth M, Handley OJ, Schwenke C, et al. Observing Huntington's disease: the european Huntington's disease Network's REGISTRY. PLoS Curr. 2010;2: RRN1184.

19. Alternatives for reducing chorea in Huntington disease, a long-term trial (ARC-HD): Updated week 8 results for the switch cohort [abstract]. Mov Disord. 2016;31:(2).

20. Epping EA, Kim Jl, Craufurd D, et al. Longitudinal psychiatric symptoms in prodromal Huntington's disease: a decade of data. Am J Psychiatry. 2016; 173:184-92.

21. Kenney C, Hunter C, Mejia N, Jankovic J. Is history of depression a contraindication to treatment with tetrabenazine? Clin Neuropharmacol. 2006;29:259-64

22. Armstrong MJ, Miyasaki JM, American Academy of N. Evidence-based guideline: pharmacologic treatment of chorea in Huntington disease: report of the guideline development subcommittee of the American academy of neurology. Neurology. 2012;79:597-603.

23. Paulsen JS, Nehl C, Hoth KF, et al. Depression and stages of Huntington's disease. J Neuropsychiatry Clin Neurosci. 2005;17:496-502.

\section{Submit your next manuscript to BioMed Central and we will help you at every step:}

- We accept pre-submission inquiries

- Our selector tool helps you to find the most relevant journal

- We provide round the clock customer support

- Convenient online submission

- Thorough peer review

- Inclusion in PubMed and all major indexing services

- Maximum visibility for your research

Submit your manuscript at www.biomedcentral.com/submit
Biomed Central 